\title{
Shape transition in some rare-earth nuclei in relativistic mean field theory
}

\author{
B. K. Agrawal ${ }^{1 *}$, Tapas $\mathrm{Sil}^{2}$, S. K. Samaddar ${ }^{1}$ and J. N. De ${ }^{2}$ \\ (1) Saha Institute of Nuclear Physics, 1/AF, Bidhannagar, Calcutta - 700064, India \\ (2) Variable Energy Cyclotron Centre, 1/AF, Bidhannagar, Calcutta - 700064, India
}

(October 24, 2018)

\begin{abstract}
A systematic study of the temperature dependence of the shapes and pairing gaps of some isotopes in the rare-earth region is made in the relativistic Hartree-BCS theory. Thermal response to these nuclei is always found to lead to a phase transition from the superfluid to the normal phase at a temperature $T_{\Delta} \sim 0.4-0.8 \mathrm{MeV}$ and a shape transition from prolate to spherical shapes at $T_{c} \sim 1.0-2.5 \mathrm{MeV}$. These shape transition temperatures are appreciably higher than the corresponding ones calculated in the non-relativistic framework with the pairing plus quadrupole interaction. Study of nuclei with continued addition of neutron pairs for a given isotope shows that with increased ground state deformation, the transition to the spherical shape is delayed in temperature. A strong linear correlation between $T_{\Delta}$ and the ground state pairing gap $\Delta^{0}$ is observed; a well-marked linear correlation between $T_{c}$ and the ground state quadrupole defromation $\beta_{2}^{0}$ is also seen. The thermal evolution of the hexadecapole deformation is further presented in the paper.
\end{abstract}

PACS numbers: 21.10.Ma, 21.60.-n, 27.70.+q

*E-mail:bijay@tnp.saha.ernet.in 


\section{INTRODUCTION}

Heating can have a profound effect on nuclear shapes, causing a variety of shape transitions. Experimentally, such responses to the thermal excitations have been studied from the shapes of the giant dipole resonances(GDR) built on excited states [1] 3]. Theoretically, they have been studied earlier in a finite temperature non-relativistic microscopic Hartree-Fock [4.5] and Hartree-Fock Bogoliubov (HFB) framework [6 9] with a pairing plus quadrupole $(\mathrm{P}+\mathrm{Q})$ interaction. For the nuclei studied, it has been found that while the superfluid nuclear phase has transition to the normal phase typically at $\mathrm{T} \sim 0.5 \mathrm{MeV}$, the deformed shapes have transition to the spherical ones at a higher temperature, mostly between $\mathrm{T} \sim 1.0$ to 1.8 $\mathrm{MeV}$ for rare-earth nuclei. These calculations, however, have some limitations; they employ a simplistic model Hamiltonian in a limited model space, an inert core is assumed, moreover, the Coulomb interaction has not been taken into account realistically. The understanding of the universal pattern of the mean field shape evolution with temperature has also been tried in a macroscopic approach [10,11] commonly referred to as the Landau theory of phase transition. A quantitative estimate of the persistence of the ground state deformation [12] with temperature is however seen to be missing in some cases.

Recently, we have undertaken a study [13] of the thermal evolution of nuclear properties, particularly the phase transition in the nuclear shape and the superfluidity in the relativistic mean field (RMF) theory. The pairing effects have been included in the BCS approximation. The RMF theory [14 16] has proved to be an extremely powerful tool in explaining the gross properties of nuclei over the entire periodic table. In contrast to the non-relativistic models, this theory employs a single set of parameters to explain all these properties. Moreover, in such calculations, the model space used is sufficiently large and all the nucleons are treated on equal footing. The calculations reported in Ref. [13 are performed for only two

rare-earth nuclei, namely, ${ }^{166} \mathrm{Er}$ and ${ }^{170} \mathrm{Er}$. It is found that the phase transition for the nuclear shape from the prolate to the spherical occurs at a temperature significantly higher than that obtained in the $(\mathrm{P}+\mathrm{Q})$ model and that the transition is relatively smooth. A very 
recent calculation by Egido et al [17] in a nonrelativistic approach but with the realistic Gogny force reveals that the characteristics of the nuclear shape transition are very similar to those obtained in the RMF theory. We have therefore undertaken a more systematic study of the shape transition for the rare-earth nuclei in the relativistic mean field theory in the present paper. For this purpose, we have considered various even-even isotopes of $S m$, $G d$ and Dy. A more quantitative study, particularly of the shape transitions, calls for the inclusion of thermal fluctuations [13, 18, 19]. However, this is too computer intensive and are not included in the present paper.

The organization of the paper is as follows: we discuss the theoretical framework briefly in section II. The results and discussions are presented in section III and the concluding remarks are given in section IV.

\section{FORMALISM}

We employ the nonlinear $\sigma-\omega-\rho$ version of the RMF theory [15]. The Lagrangian density for the nucleon-meson many body system is taken as

$$
\begin{aligned}
\mathcal{L}= & \bar{\Psi}_{i}\left(i \gamma^{\mu} \partial_{\mu}-M\right) \Psi_{i}+\frac{1}{2} \partial^{\mu} \sigma \partial_{\mu} \sigma-U(\sigma)-g_{\sigma} \bar{\Psi}_{i} \sigma \Psi_{i} \\
& -\frac{1}{4} \Omega^{\mu \nu} \Omega_{\mu \nu}+\frac{1}{2} m_{\omega}^{2} \omega^{\mu} \omega_{\mu}-g_{\omega} \bar{\Psi}_{i} \gamma^{\mu} \omega_{\mu} \Psi_{i}-\frac{1}{4} \vec{R}^{\mu \nu} \vec{R}_{\mu \nu}+\frac{1}{2} m_{\rho}^{2} \vec{\rho}^{\mu} \vec{\rho}_{\mu} \\
& -g_{\rho} \bar{\Psi}_{i} \gamma^{\mu} \vec{\rho}_{\mu} \vec{\tau} \Psi_{i}-\frac{1}{4} F^{\mu \nu} F_{\mu \nu}-e \bar{\Psi}_{i} \gamma^{\mu} \frac{\left(1-\tau_{3}\right)}{2} A_{\mu} \Psi_{i} .
\end{aligned}
$$

The arrows indicate isovector quantities. The mesons included in the description are the $\sigma, \omega$ and $\rho$ mesons. For an appropriate description of the nuclear surfaces [20], a non-linear scalar self-interaction term $U(\sigma)$ of the $\sigma$ meson is included in the Lagrangian

$$
U(\sigma)=\frac{1}{2} m_{\sigma}^{2} \sigma^{2}+\frac{1}{3} g_{2} \sigma^{3}+\frac{1}{4} g_{3} \sigma^{4}
$$

The meson masses are given by $m_{\sigma}, m_{\omega}$ and $m_{\rho}$, the nucleon mass is $M$ and $g_{\sigma}, g_{\omega}, g_{\rho}$ and $e^{2} / 4 \pi=1 / 137$ are the coupling constants for the mesons and the photon. The field tensors for the vector mesons $\omega$ and $\rho$ are given by $\Omega^{\mu \nu}$ and $\vec{R}^{\mu \nu}$, for the electromagnetic 
field, it is $F^{\mu \nu}$. Recourse to variational principle followed by the mean field approximation treating the fields as $c$ - numbers results in the Dirac equation for the nucleon and the KleinGordon type equations for the mesons and the photon. For the static case, along with the time-reversal invariance and charge conservation, the equations get simplified. The resulting equations, known as RMF equations, have the following form. The Dirac equation for the nucleon is

$$
\{-i \alpha \cdot \nabla+V(\mathbf{r})+\beta[M+S(\mathbf{r})]\} \Psi_{i}=\epsilon_{i} \Psi_{i}
$$

where $V(\mathbf{r})$ represents the vector potential

$$
V(\mathbf{r})=g_{\omega} \omega_{0}(\mathbf{r})+g_{\rho} \tau_{3} \rho_{0}(\mathbf{r})+e \frac{\left(1-\tau_{3}\right)}{2} A_{0}(\mathbf{r}),
$$

and $S(\mathbf{r})$ is the scalar potential

$$
S(\mathbf{r})=g_{\sigma} \sigma(\mathbf{r})
$$

which contributes to the effective mass as

$$
M^{*}(\mathbf{r})=M+S(\mathbf{r})
$$

The Klein-Gordon equations for the mesons and the electromagnetic fields with the nucleon densities as sources are

$$
\begin{aligned}
\left\{-\Delta+m_{\sigma}^{2}\right\} \sigma(\mathbf{r}) & =-g_{\sigma} \rho_{s}(\mathbf{r})-g_{2} \sigma^{2}(\mathbf{r})-g_{3} \sigma^{3}(\mathbf{r}) \\
\left\{-\Delta+m_{\omega}^{2}\right\} \omega_{0}(\mathbf{r}) & =g_{\omega} \rho_{v}(\mathbf{r}) \\
\left\{-\Delta+m_{\rho}^{2}\right\} \rho_{0}(\mathbf{r}) & =g_{\rho} \rho_{3}(\mathbf{r}) \\
-\Delta A_{0}(\mathbf{r}) & =e \rho_{c}(\mathbf{r}) .
\end{aligned}
$$

The corresponding densities are

$$
\begin{aligned}
\rho_{s} & =\sum_{i} n_{i} \bar{\Psi}_{i} \Psi_{i}, \\
\rho_{v} & =\sum_{i} n_{i} \Psi_{i}^{\dagger} \Psi_{i}, \\
\rho_{3} & =\sum_{i} n_{i} \Psi_{i}^{\dagger} \tau_{3} \Psi_{i}, \\
\rho_{c} & =\sum_{i} n_{i} \Psi_{i}^{\dagger} \frac{\left(1-\tau_{3}\right)}{2} \Psi_{i} .
\end{aligned}
$$


Here the sums are taken over the particle states only, i.e., the negative-energy states are neglected. The partial occupancy $\left(n_{i}\right)$ at finite temperature in the BCS approximation is

$$
n_{i}=\frac{1}{2}\left[1-\frac{\epsilon_{i}-\lambda}{\tilde{\epsilon}_{i}}\left(1-2 f\left(\tilde{\epsilon}_{i}, T\right)\right)\right]
$$

with $f\left(\tilde{\epsilon}_{i}, T\right)=1 /\left(1+e^{\tilde{\epsilon}_{i} / T}\right) ; \tilde{\epsilon}_{i}=\sqrt{\left(\epsilon_{i}-\lambda\right)^{2}+\Delta^{2}}$ is the quasiparticle energy where $\epsilon_{i}$ is the single-particle energy for the state $i$. The chemical potential $\lambda$ for protons (neutrons) is obtained from the requirement

$$
\sum_{i} n_{i}=Z(N)
$$

The sum is taken over proton (neutron) states. The gap parameter $\Delta$ is obtained by minimising the free energy

$$
F=E-T S,
$$

where

$$
E(T)=\sum_{i} \epsilon_{i} n_{i}+E_{\sigma}+E_{\sigma N L}+E_{\omega}+E_{\rho}+E_{C}+E_{\text {pair }}+E_{c . m .}-A M
$$

and

$$
S=-\sum_{i}\left[f_{i} \ln f_{i}+\left(1-f_{i}\right) \ln \left(1-f_{i}\right)\right]
$$

with

$$
\begin{aligned}
E_{\sigma} & =-\frac{1}{2} g_{\sigma} \int d^{3} r \rho_{s}(\mathbf{r}) \sigma(\mathbf{r}), \\
E_{\sigma N L} & =-\frac{1}{2} \int d^{3} r\left\{\frac{1}{3} g_{2} \sigma^{3}(\mathbf{r})+\frac{1}{2} g_{3} \sigma^{4}(\mathbf{r})\right\}, \\
E_{\omega} & =-\frac{1}{2} g_{\omega} \int d^{3} r \rho_{v}(\mathbf{r}) \omega^{0}(\mathbf{r}), \\
E_{\rho} & =-\frac{1}{2} g_{\rho} \int d^{3} r \rho_{3}(\mathbf{r}) \rho^{0}(\mathbf{r}), \\
E_{C} & =-\frac{e^{2}}{8 \pi} \int d^{3} r \rho_{C}(\mathbf{r}) A^{0}(\mathbf{r}), \\
E_{\text {pair }} & =-\frac{\Delta^{2}}{G} \\
E_{c . m .} & =-\frac{3}{4} \hbar \omega_{0}=-\frac{3}{4} 41 A^{-1 / 3} .
\end{aligned}
$$


Here $G$ and $A$ are the pairing strength and the mass number respectively. The single-particle energies and the fields appearing in eqs. (15) - (21) are obtained from the self-consistent solution of eqs. (3) - (10). The temperature dependent occupancies of the fermions induce temperature dependence in the bosonic fields through the source terms as seen from eqs. $(\sqrt{7})-(10)$

We generate these self-consistent solutions using the basis expansion method [15,21 ; this yields the quadrupole deformation $\beta_{2}$, the hexadecapole deformation $\beta_{4}$ and the proton and neutron pairing gaps $\Delta_{p}$ and $\Delta_{n}$ as a function of temperature.

\section{RESULTS AND DISCUSSIONS}

We have chosen even-even isotopes of the nuclei $S m, G d$ and $D y$ for the study of the pairing and shape transitions. The NL3 parameter set is chosen for the values of the coupling constants and the masses of the mesons and the nucleons. This parameter set reproduces best the ground state as well as the compression properties of finite nuclei simultaneously [22]; however, it has already been reported in Ref. [13] that the results for shape transition are not that sensitive to the choice of the parameter set. The pairing gaps $\Delta_{n}^{0}$ and $\Delta_{p}^{0}$ for neutrons and protons for a nucleus in the ground state are determined from the experimental oddeven mass differences [23]. The single-particle states are calculated using spherical oscillator basis with twelve shells. The values of the chemical potential and the pairing gap at a given temperature are determined using all the single particle states upto $2 \hbar \omega_{0}$ (the model space) above the Fermi surface without assuming any core.

At finite temperature, because of the partial occupancies of nucleons above the Fermi surface, it is in principle necessary to have a larger basis and an extended model space. It is further necessary to take effects due to continuum into account [24]. In order to check the convergence of the calculations, we have enlarged the basis space from twelve shells to twenty shells and have extended the model space to include single-particle states upto $3 \hbar \omega_{0}$ above the Fermi surface. For this extended model space, the pairing strength $G$ is adjusted 
to reproduce the ground state pairing gap. The changes in the values of the observables are found to be insignificant due to this extension of the basis and model space even at the highest temperature of our interest $(T \sim 3.0 \mathrm{MeV})$. To estimate the importance of the continuum corrections on the observables we report here, we calculated the occupancy $n^{(+)}$ of the single particle states with positive energy. For $T<1 \mathrm{MeV}$, practically there is no particle in the positive energy states $\left(n^{(+)}=0\right)$ and at the highest temperature of interest studied here $(T=2.7 \mathrm{MeV}), n^{(+)} / A=0.011$ which is very small. It is therefore expected that continuum corrections may not play an important role in the temperature range we study. Calculations of nuclear level density in earlier studies [13,25], have shown that the continuum corrections are not important for $T$ upto $\sim 3 \mathrm{MeV}$. The continuum effects may grow stronger for $T>3 \mathrm{MeV}$, however, this is beyond the shape transition temperatures and so we have not taken this into account.

The temperature evolution of the quadrupole deformation $\beta_{2}$ with neutron number $N=$ 86 and 88 for the systems $S m, G d$ and $D y$ are displayed in Fig. 1. It is well known that addition of nucleons beyond the closed shell gives nuclei progressive prolate deformation upto around the middle of the next shell closure. This is reflected in the figure for all the isotopes and isotones. It is also seen that the critical temperature increases with addition of nucleons for these systems. It is not immediately apparent whether there is a close correlation between the ground state deformation $\beta_{2}^{0}$ and the critical temperature $T_{c}$ for shape transition; we come back to this issue later. In the top panel of this figure, particularly for ${ }^{148} \mathrm{Sm}$, it is seen that the deformation increases a little with temperature before finally falling to zero. This is due to the delicate balance between the temperature dependence of the pairing force and the nuclear interaction as derived from the RMF theory. The dramatic buildup of a deformation in a temperature window for this nucleus as seen earlier [6,7] in the non-relativistic framework is absent in our calculations.

The temperature dependence of the pairing gaps $\Delta_{p}$ and $\Delta_{n}$ for protons and neutrons for the two isotopes each of $S m, G d$ and $D y$ are shown in Figs. 2 and 3. The pairing gaps decrease monotonically with temperature, vanishing at $T \sim 0.6-0.7 \mathrm{MeV}$ for neutrons and 
at $\sim 0.65-0.85 \mathrm{MeV}$ for protons. The sudden collapse of the pairing gap and the nuclear deformation at some specified temperatures signifies phase transitions; these correspond to transition from the superfluid nuclear phase to the normal phase and a transition from the deformed shape to the spherical shape, respectively. These transitions show up as bumps (displayed in Fig. 4 for ${ }^{148} \mathrm{Sm}$ and ${ }^{150} \mathrm{Sm}$ ) in the temperature evolution of the heat capacity defined as

$$
C(T)=\frac{\partial E^{*}}{\partial T}
$$

where $E^{*}$ is the excitation energy of the nucleus in question. At a temperature $T_{\Delta} \sim 0.6$ $\mathrm{MeV}$, the twin peaks are seen for both the nuclei referring to the dissolution of the neutron and proton pairing gaps. These are the characteristic signatures of second order phase transition from the superfluid to the normal phase. A somewhat more prominent bump is seen at a temperature $T_{c} \sim 1.15 \mathrm{MeV}$ for ${ }^{148} \mathrm{Sm}$ (upper panel). This corresponds to the nuclear shape transition. Addition of two neutrons (lower panel for ${ }^{150} \mathrm{Sm}$ ) shifts the shape transition temperature to $T_{c} \sim 1.6 \mathrm{MeV}$. This is possibly due to the larger ground state deformation of the ${ }^{150} \mathrm{Sm}$ nucleus.

From the study of the hot ${ }^{148} \mathrm{Sm}$ and ${ }^{150} \mathrm{Sm}$ nuclei, it was conjectured earlier [7] that addition of two neutrons might increase the critical temperature for deformation collapse. To test this conjecture in detail, we have calculated the ground state quadrupole deformation and shape transition temperatures for a host of even-even $S m$ isotopes. The results are displayed in Fig. 5. In the vicinity of the closed shell $(N=82)$, the ground state quadrupole deformation $\beta_{2}^{0}$ increases fast with the addition of two neutrons as seen from the top panel of Fig. 5. As the neutron number approaches the mid shell, the deformation levels off, and then, as is well known, switches over to the oblate shape [26] (not shown in the figure). The critical temperature $\left(T_{c}\right)$ for the shape transition also increases with neutron pair addition as seen in the bottom panel of Fig. 5. The functional behavior of $\beta_{2}^{0}$ and $T_{c}$ with neutron number $N$ are found to be very similar. Indeed there is a strong correlation between $\beta_{2}^{0}$ and $T_{c}$ as displayed in Fig. 6. The filled circles refer to the results from the present calculation; 
they can be fairly well fitted with a straight line

$$
T_{c}=7.75 \beta_{2}^{0}
$$

The fit is obtained using results from the twelve different isotopes of $S m, G d, D y$ and $E r$ nuclei. The results calculated [7,8, 27] in the $(\mathrm{P}+\mathrm{Q})$ model are also presented in the figure (open squares) for a comparison with those obtained from the RMF theory. The $T_{c}-\beta_{2}^{0}$ correlation is then also found to be approximately linear with a smaller slope. The shape transition temperatures obtained in the present calculations are somewhat higher compared to those obtained in the $(P+Q)$ model; one may be inclined to attribute this difference to the higher effective mass in the $(P+Q)$ model. However, calculations with different sets of field parameters in the RMF theory with considerably different values of the effective masses yield conflicting results. We have done calculations for ${ }^{150} \mathrm{Sm}$ with the parameter sets HS and NL2 which yield very different $M^{*} / M$ [15] (0.54 and 0.67), but the $T_{c}$ comes out to be 1.45 and $1.75 \mathrm{MeV}$, respectively, contrary to the simple-minded expectations. Therefore no simple explanation for the higher values of $T_{c}$ in the RMF theory is obvious.

How does the pairing transition temperature $T_{\Delta}$ depend on the pairing gap at $T=0$ ? Intuitively one would expect the collapse of nuclear superfluidity at a larger temperature if the ground state pairing gap $\Delta^{0}$ is larger. To study it quantitatively, we have done calculations for the different isotopes of $S m$. The results are shown in Fig. 7. In its upper panel, we display the experimental neutron and proton ground state pairing gaps as a function of neutron number for the $S m$ isotopes; in the lower panel, the pairing transition temperatures $T_{\Delta}$ are displayed. An extremely strong correlation between $T_{\Delta}$ and $\Delta^{0}$ for both neutrons and protons is seen; this is manifest in the linear relationship

$$
T_{\Delta_{p}}=0.56 \Delta_{p}^{0}, \quad T_{\Delta_{n}}=0.60 \Delta_{n}^{0}
$$

for both neutrons and protons which is also shown in Fig.8. The points in the figure include, in addition to $S m$, results from $G d, D y$ and $E r$ isotopes. The relation between $T_{\Delta}$ and $\Delta^{0}$ is in very close agreement to that obtained in the nonrelativistic $(\mathrm{P}+\mathrm{Q})$ model [7]. 
The hexadecapole deformation $\beta_{4}$, if any, also collapses at the same shape transition temperature $T_{c}$ as the quadrupole deformation. In Fig. 9, we plot the hexadecapole moment (a measure of $\beta_{4}$ ) as a function of temperature for ${ }^{148} \mathrm{Sm}$ and ${ }^{150} \mathrm{Sm}$. Addition of neutrons

gives larger ground state $\beta_{4}^{0}$. The deformation increases smoothly at low temperature upto $T \sim 0.7 \mathrm{MeV}$ and then collapses to zero at $T_{c}$. The initial enhancement of this deformation is related to the weakening of the pairing correlations with temperature. Such an enhancement is also seen in a finite temperature Hartree-Fock Bogoliubov (FTHFB) calculation using the finite range density-dependent Gogny force [17].

\section{CONCLUSIONS}

The relativistic mean field theory has been applied to understand properties of some rareearth even-even nuclei at finite temperature. Pairing effects have been included through the BCS approximation. Focus is made mainly on the temperature-induced transition from the nuclear superfluid phase to the normal phase and also on the shape transition from a deformed shape to a spherical one. To find out the systematics of the dependence of the pairing and shape transition temperatures on the values of the ground state pairing gap and the ground state deformation, we have done calculations for several isotopes of different rare-earth nuclei. We find that there is a linear correlation between the above mentioned transition temperatures and the equilibrium values of the pairing gap and deformation at zero temperature. The linear relationship is extremely good for the pairing gap and quite fair for the deformation. In the range of nuclei that are studied here, it is indeed possible to estimated very closely the value of the pairing transition temperature $T_{\Delta}$ for both neutrons and protons, as the ground state pairing gap can be calculated from the systematics of binding energy. Similarly, it appears that the shape transition temperatures can be well estimated since the ground state quadrupole deformations can be experimentally extracted. The transition temperatutres $T_{\Delta}$ are not too different from those calculated earlier in a nonrelativistic framework; the shape transition temperatures $T_{c}$ however seem to be higher than 
the corresponding values calculated in the $(\mathrm{P}+\mathrm{Q})$ model. These higher values of the shape transition temperatures are however found to be very compatible with the ones obtained from the realistic Gogny force. 


\section{REFERENCES}

[1] K. A. Snover, Annu. Rev. Nucl. Part. Sc. 36, 545 (1986).

[2] J. J. Gaardhoje, Annu. Rev. Nucl. Part. Sc. 42, 483 (1992).

[3] V. Nanal et al, Nucl. Phys. A649, 153c (1999).

[4] M. Brack and P. Quentin, Phys. Scr. A10, 163 (1974).

[5] P. Quentin and H. Flocard, Annu. Rev. Nucl. Part. Sc. 28, 523 (1978).

[6] A. L. Goodman, Phys. Rev. C33, 2212 (1986).

[7] A. L. Goodman, Phys. Rev. C34, 1942 (1986).

[8] A. L. Goodman, Phys. Rev. C38, 977 (1988).

[9] J. L. Egido, P. Ring and H. J. Mang, Nucl. Phys. A451, 77 (1986).

[10] S. Levit and Y. Alhassid, Nucl. Phys. A413, 439 (1984).

[11] Y. Alhassid, J. Zingman and S. Levit, Nucl. Phys. A469, 205 (1987).

[12] M. P. Kelley, K. A. Snover, J. P. S. van Schagen, M. Kicinska- Haboir and Z. Trznadel, Phys. Rev. Lett. 82, 3404 (1999).

[13] B. K. Agrawal, Tapas Sil, J. N. De and S. K. Samaddar, Phys. Rev. C62, 044307 (2000).

[14] B. D. Serot and J. D. Walecka, Adv. Nucl. Phys. 16, 1 (1986).

[15] Y. K. Gambhir, P. Ring and A. Thimet, Ann. Phys. (N.Y.) 198, 132 (1990).

[16] P. Ring, Prog. Part. Nucl. Phys. 37, 193(1996).

[17] J. L. Egido, L. M. Roblado and V. Martin, Phys. Rev. Lett. 85, 26 (2000).

[18] Y. Alhassid and J. Zingman, Phys. Rev. C30, 684 (1984).

[19] R. Rossignoli and P. Ring, Ann. Phys. (N.Y) 235, 350 (1994). 
[20] J. Boguta and A. R. Bodmer, Nucl. Phys. A292, 413 (1977).

[21] P. Ring, Y. K. Gambhir and G. A. Lalazissis, Comp. Phys. Comm. 105, 77 (1997).

[22] G. A. Lalazissis, J. König and P. Ring, Phys. Rev. C55, 540 (1997).

[23] A. Bohr and B. Mottelson, Nuclear Structure, Vol. I, p. 169, W. A. Benjamin, Inc., (1969).

[24] P. Bonche, S. Levit and D. Vautherin, Nucl. Phys. A427, 278 (1984).

[25] B. K. Agrawal, S. K. Samaddar, J.N.De and S. Shlomo, Phys. Rev. C58, 3004 (1998).

[26] G. A. Lalazissis, S. Raman and P. Ring, Atomic Data and Nuclear Data Tables, 71 1, (1999).

[27] A. L. Goodman, Phys. Rev. C39, 2008 (1989). 


\section{Figure Captions}

Fig. 1 The evolution of the quadrupole deformation $\beta_{2}$ as a function of temperature for the $S m, G d$ and $D y$ isotopes.

Fig. 2 Temperature evolution of the proton pairing gap for the systems indicated.

Fig. 3 Same as in Fig. 2, but for the neutron pairing gap.

Fig. 4 Variation of heat capacity as a function of temperature for ${ }^{148} \mathrm{Sm}$ and ${ }^{150} \mathrm{Sm}$.

Fig. 5 (a) Variation of the ground state deformation $\beta_{2}^{0}$ with neutron number $N$ for the isotopes of $S m$ and (b) the shape transition temperature $T_{c}$ for different isotopes of Sm.

Fig. 6 The shape transition temperature $T_{c}$ plotted as a function of the ground state quadrupole deformation $\beta_{2}^{0}$ for different rare-earth nuclei. The full circles refer to the results from the RMF theory, the full line is the linear fit to these. The open squares refer to those from the $(\mathrm{P}+\mathrm{Q})$ model and the dashed line is the linear fit to these points.

Fig. 7 (a) The ground state neutron and proton pairing gaps as a function of neutron number for the $S m$ isotopes and (b) the neutron and proton pairing transition temperatures $T_{\Delta}$ for the different $S m$ isotopes.

Fig. 8 The correlation between the transition temperature $T_{\Delta}$ and the ground state pairing gap $\Delta^{0}$ for different nuclei in the rare-earth region.

Fig. 9 Temperature evolution of the hexadecapole moment (in units of barn ${ }^{2}$ ) for the nuclei ${ }^{148} \mathrm{Sm}$ and ${ }^{150} \mathrm{Sm}$. 


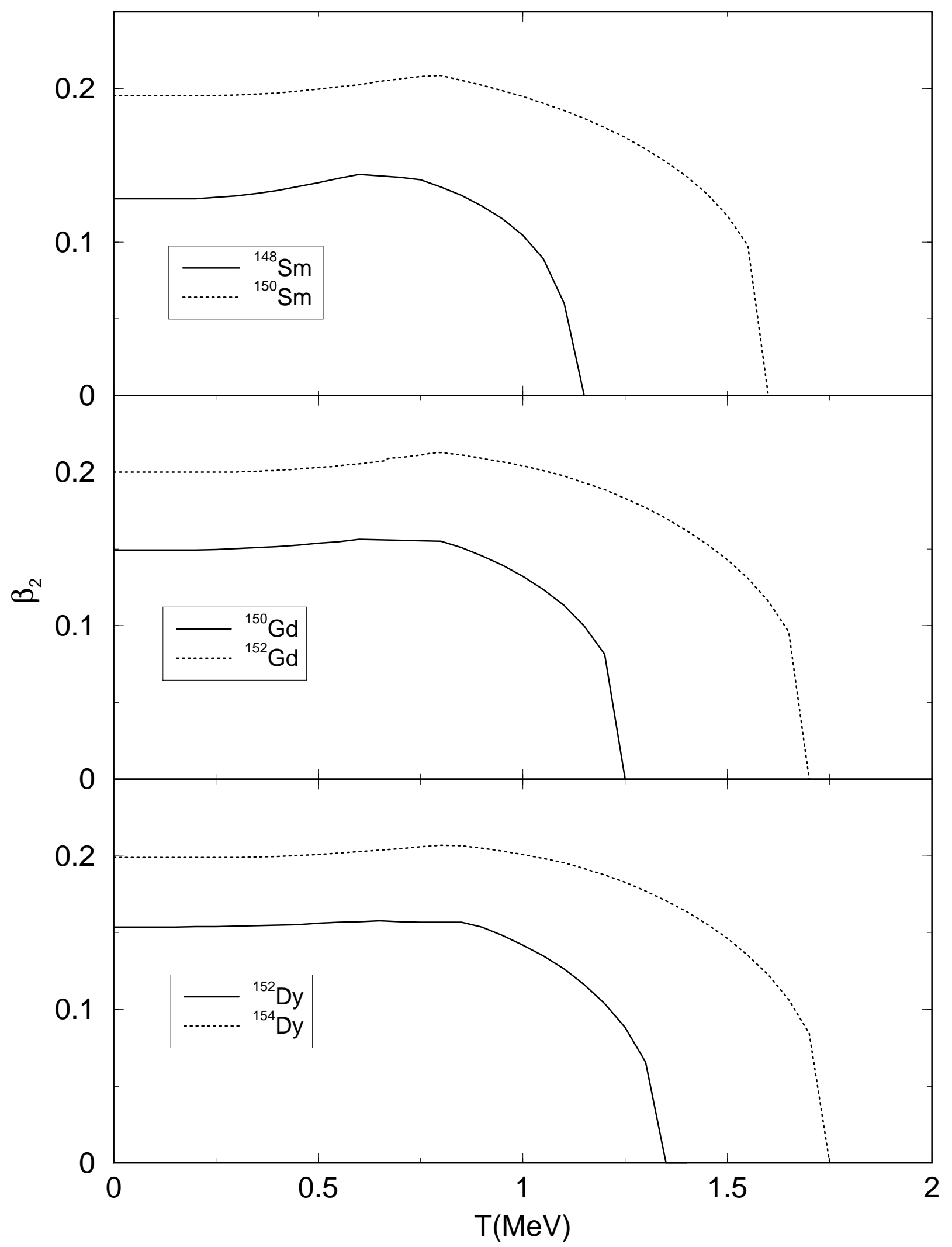

Fig. 1 


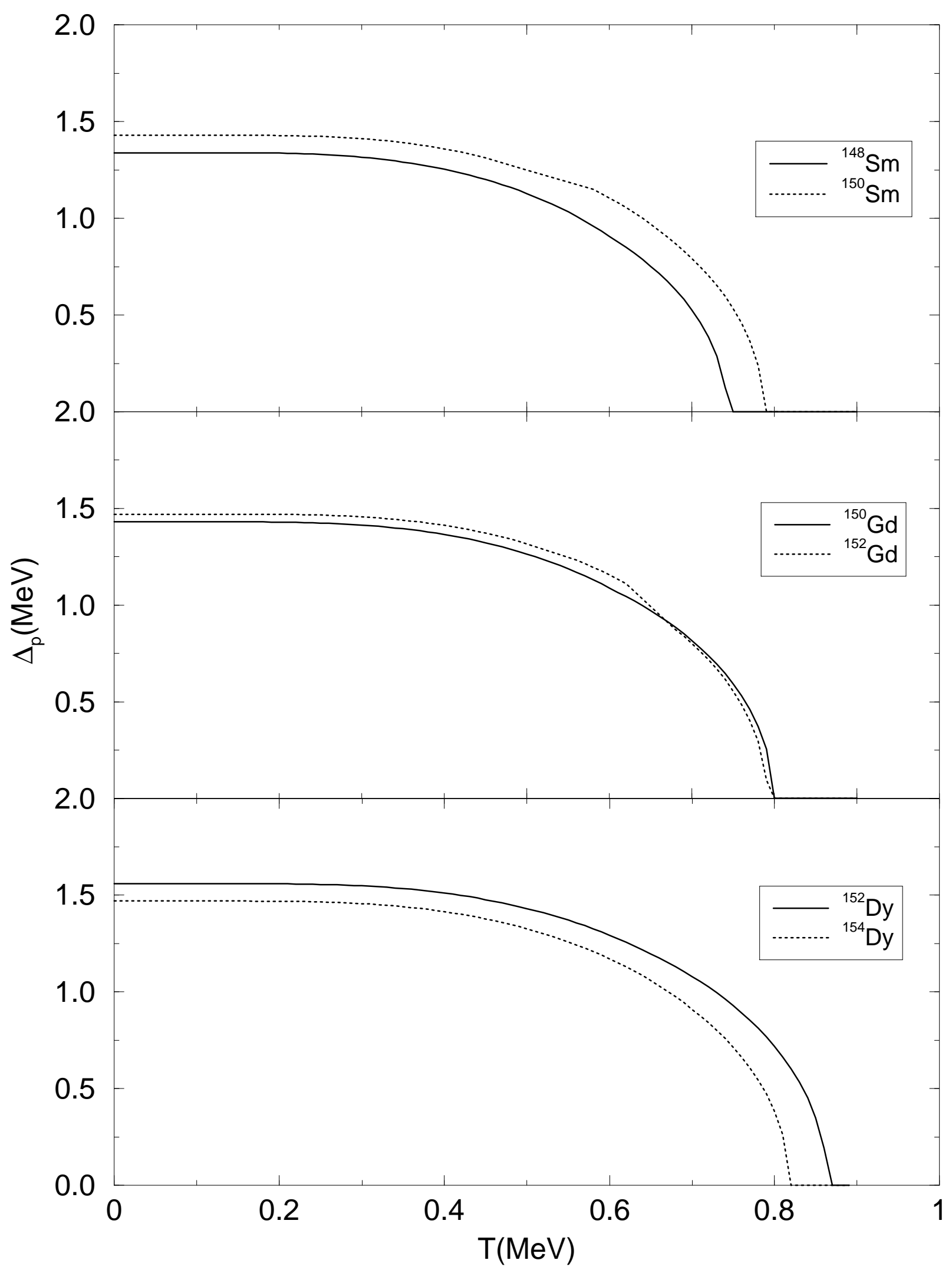

Fig. 2 


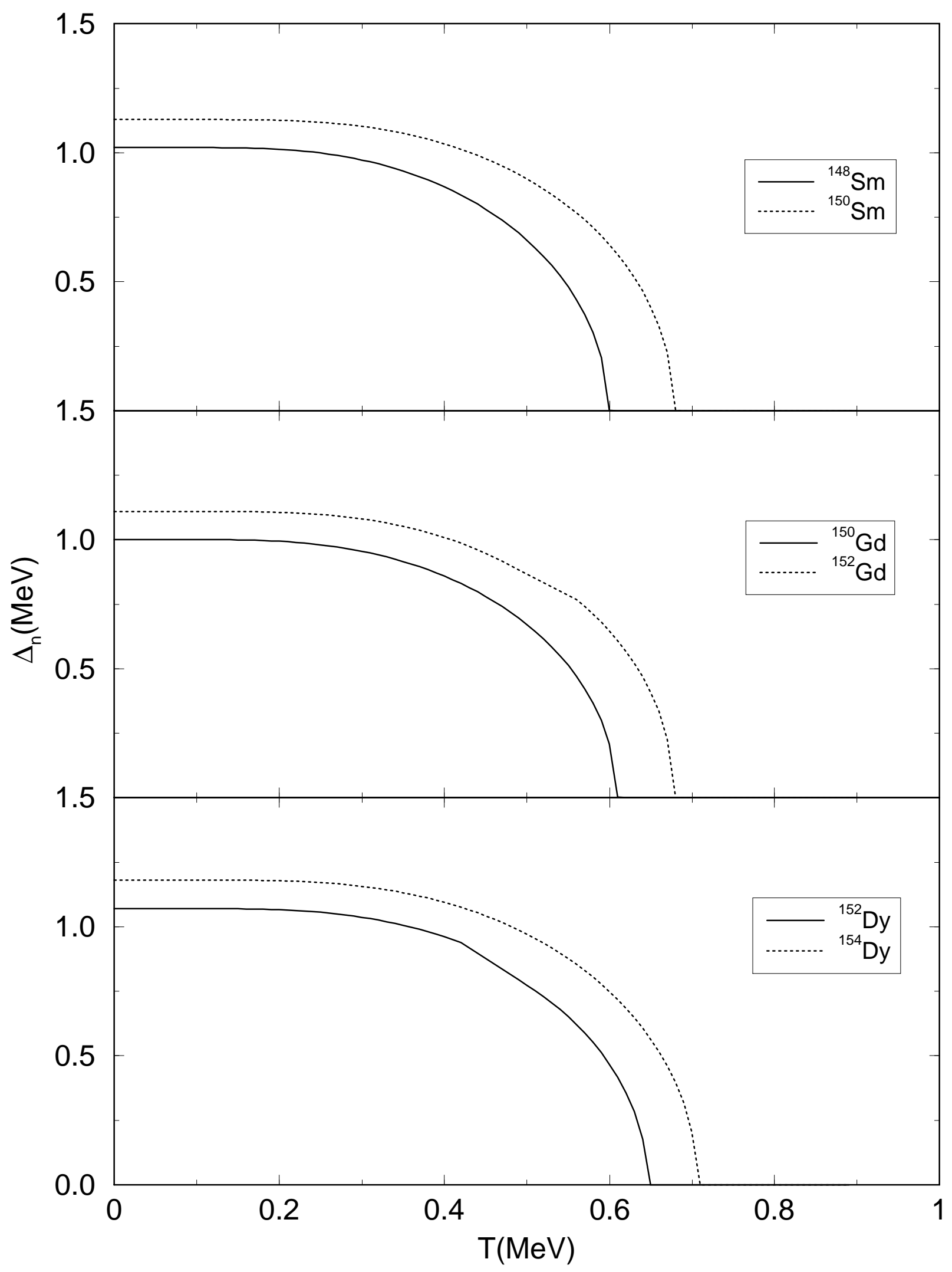

Fig. 3 


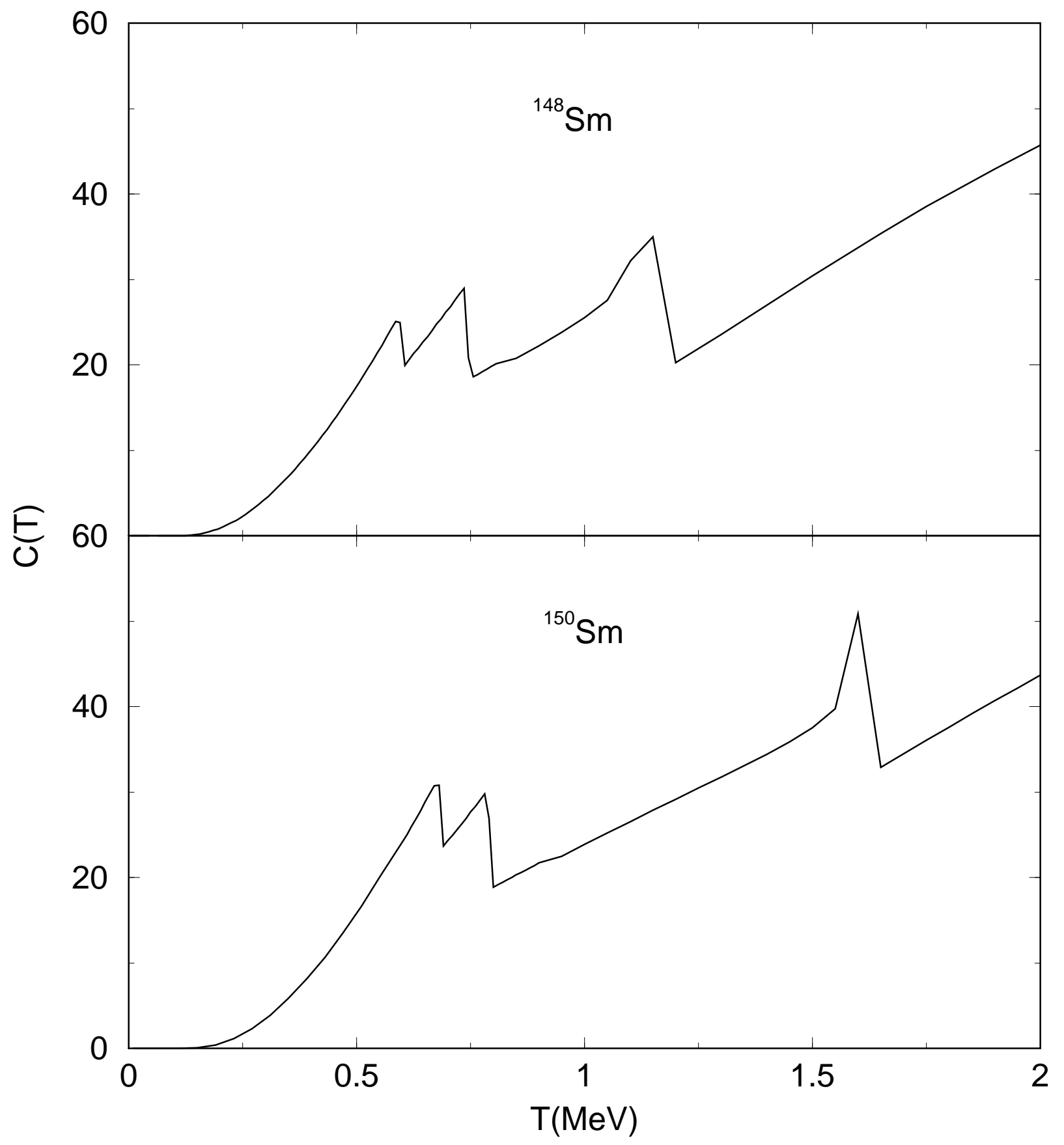

Fig. 4 


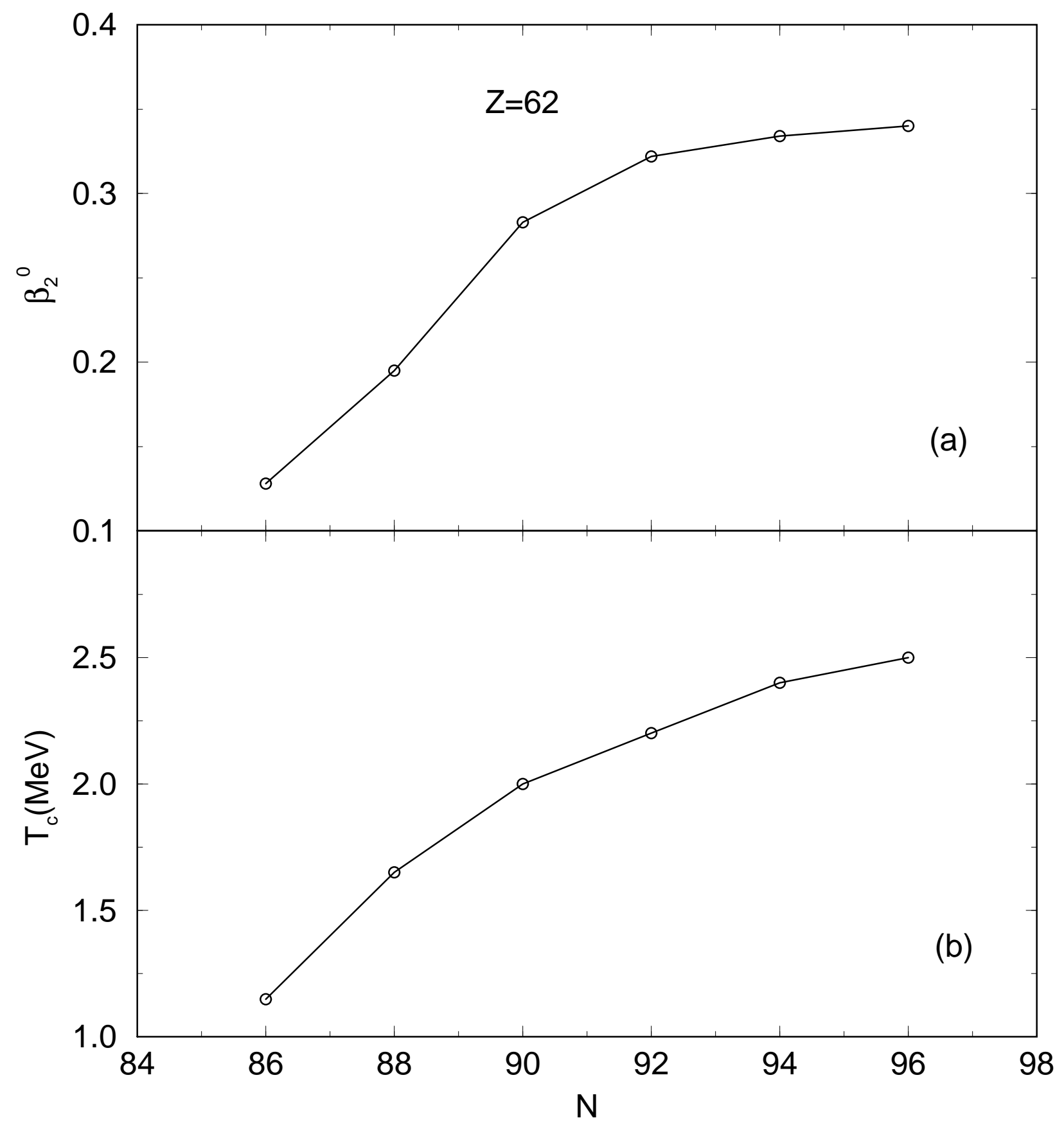

Fig. 5 


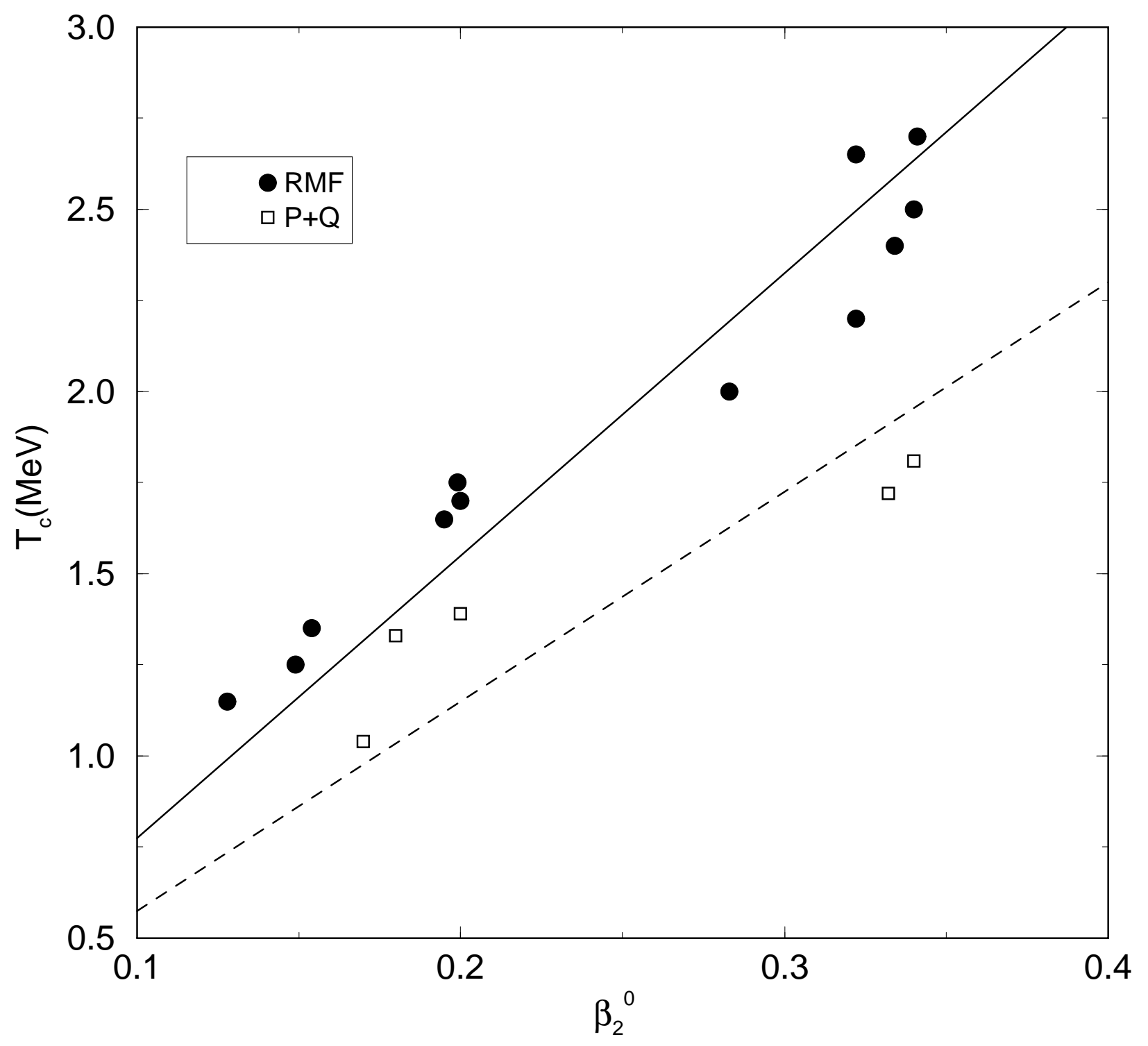

Fig. 6 


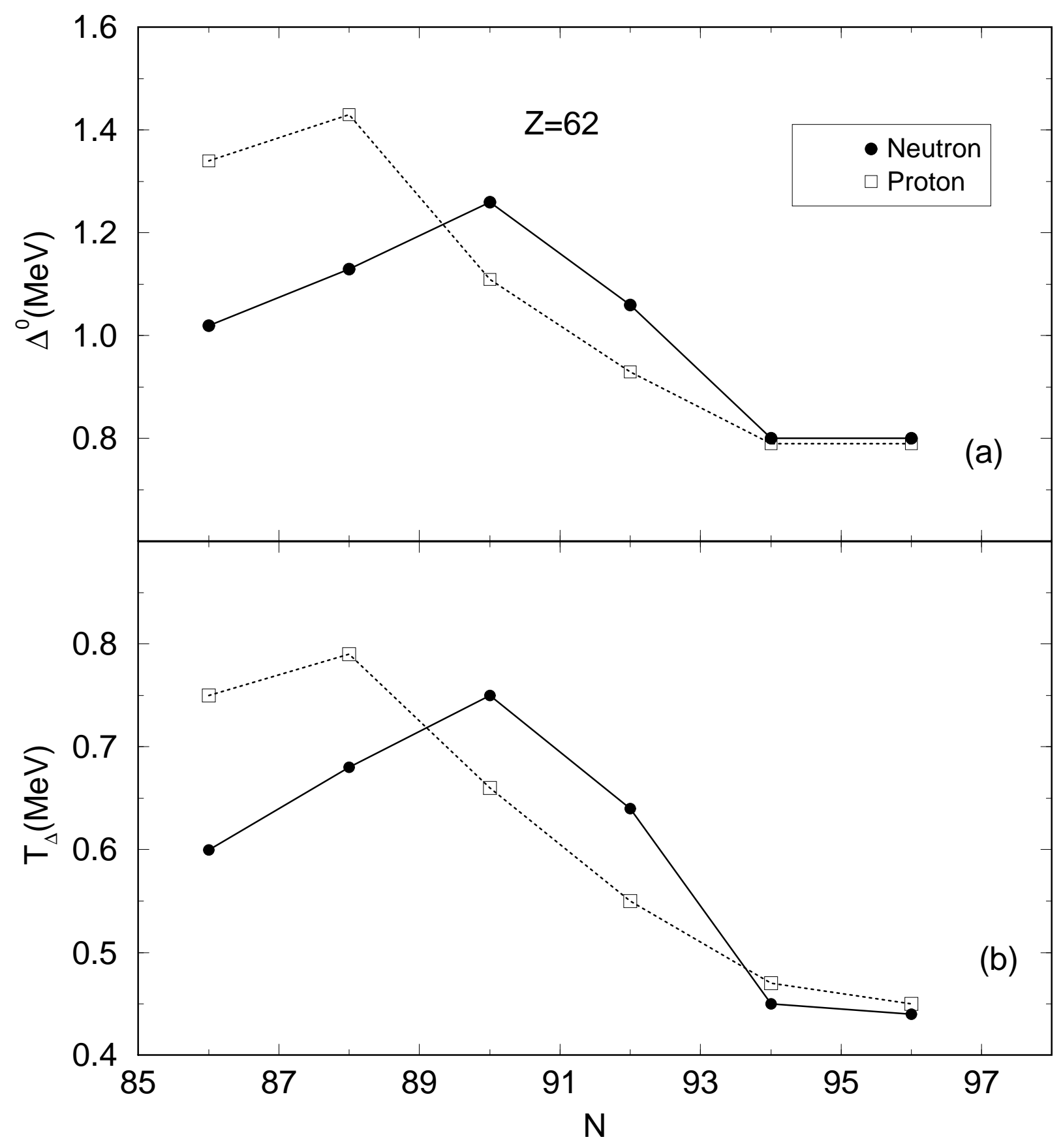

Fig. 7 


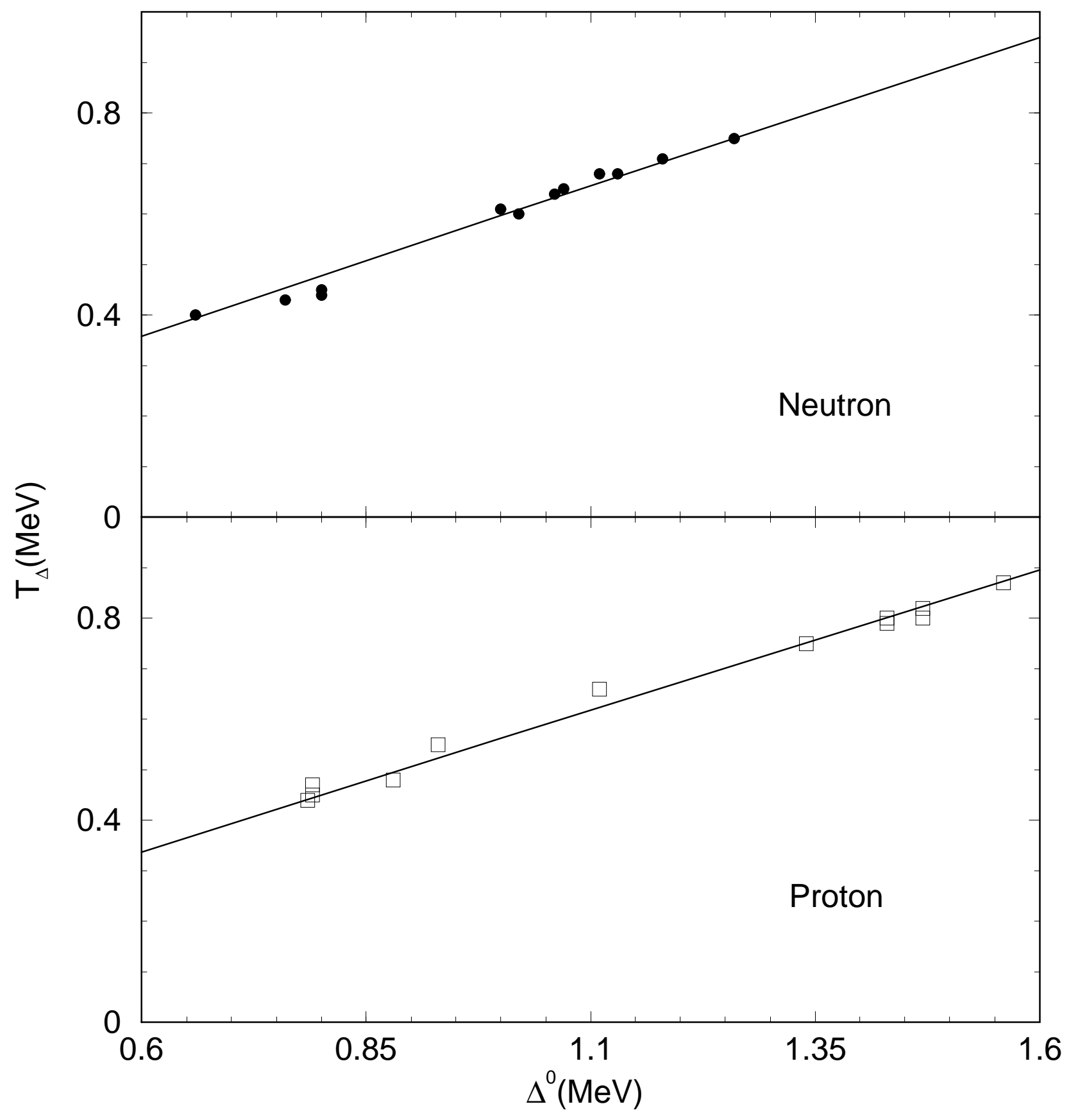

Fig. 8 


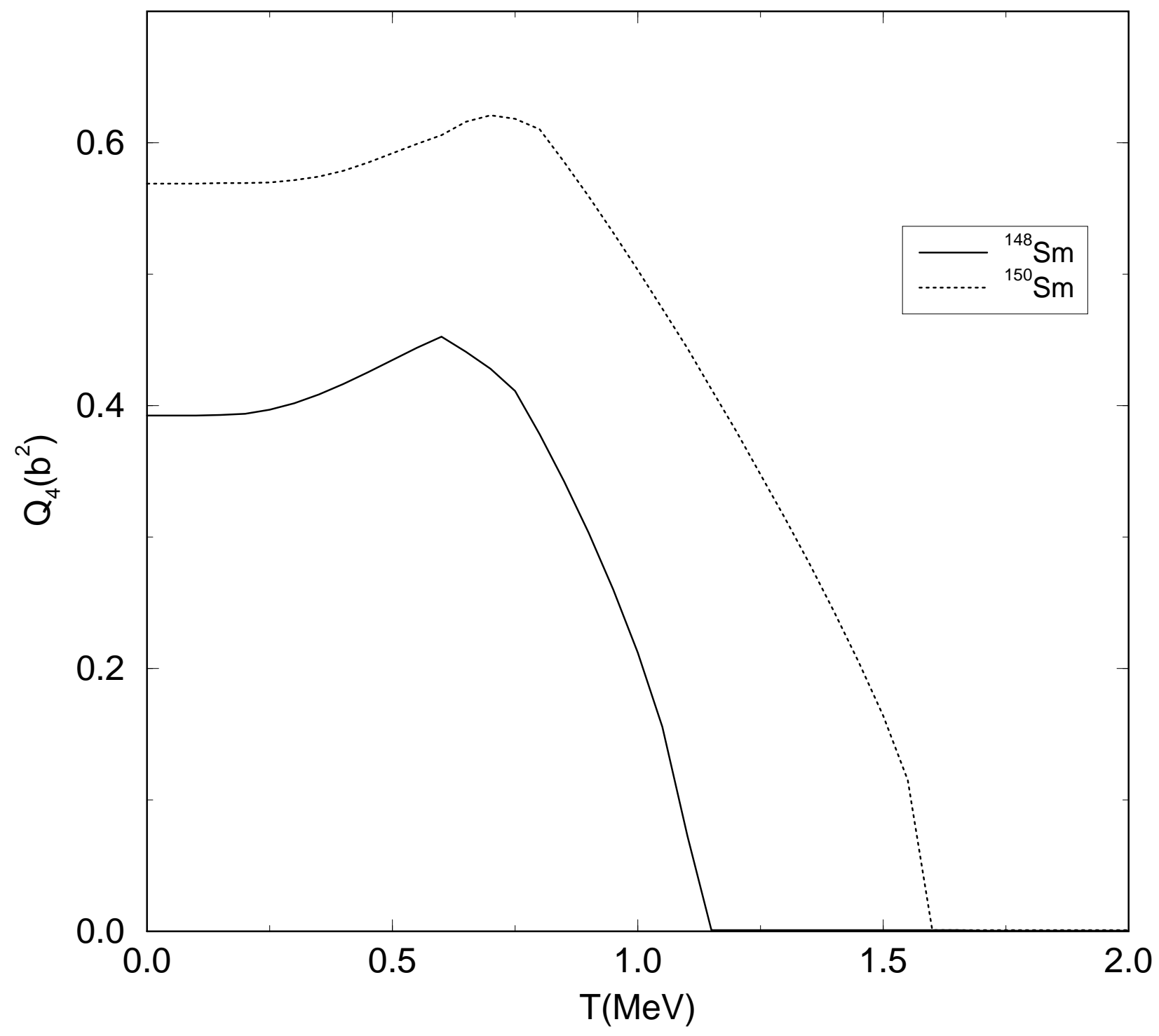

Fig. 9 